Check for updates

Cite this: Mater. Adv., 2021, 2,5150

Received 16th February 2021, Accepted 18th May 2021

DOI: $10.1039 / \mathrm{d} 1 \mathrm{ma00137j}$

rsc.li/materials-advances

\title{
An injectable serotonin-chondroitin sulfate hydrogel for bio-inspired hemostatic adhesives with high wound healing capability $\dagger$
}

\author{
Xingxia Zhang, ${ }^{\mathrm{ab}}$ Zhifang $\mathrm{Ma},{ }^{\mathrm{a}}$ Yue $\mathrm{Ke},{ }^{\mathrm{a}} \mathrm{Yu} \mathrm{Xia},{ }^{a}$ Xiaodong $\mathrm{Xu},{ }^{* \mathrm{~b}}$ Jingchuan Liu, ${ }^{\mathrm{a}}$ \\ Yumei Gong, (D) ${ }^{c}$ Qiang Shi (D) *a and Jinghua Yin ${ }^{a}$
}

\begin{abstract}
Biocompatibility, hemostatic performance and wound healing capability are key limitations for the currently available hemostatic agents. To overcome these problems, a hydrogel inspired by a platelet coagulation mediator is developed in this work as a new class of hemostatic adhesive with improved performance and wound healing capability. The hydrogel is prepared using highly biocompatible serotonin and chondroitin sulfate (CS), both of which are natural components of the body. The structural, physical and biological and hemostatic properties of the hydrogel are characterized in detail. It is demonstrated that serotonin acts as a crosslinker to form adhesive hydrogels and as a blood clotting mediator for rapid hemostasis. Chondroitin sulfate regulates cell behaviors and fates to facilitate wound healing. The serotonin-conjugated chondroitin sulfate hydrogel exhibits improved hemostatic capability in vivo and rapid wound healing after hemostasis. In addition, the wound healing capability of the hydrogel is further improved with the aloe vera powder, confirming the versatility of the hydrogel system. Therefore, chondroitin sulfate-serotonin hydrogels exhibit the potential for effective hemostasis and wound healing.
\end{abstract}

\section{Introduction}

In war and clinical trauma, massive blood loss caused by incompressible injury may lead to tissue diseases and even death. $^{1-3}$ Many hemostatic agents have been developed to manage uncontrolled bleeding. The commonly used hemostatic materials on the market include tourniquets, dressings, coagulation powders, etc. Among them, hydrogels received wide attention because of their good viscoelasticity and functionality. ${ }^{4-8}$ Particularly, in situ injectable hydrogels can closely adhere the material to irregular wounds through minimally invasive injection techniques and to perform well in humid and highly dynamic environments. ${ }^{9-13}$ However, biocompatibility, hemostatic performance and wound healing capability are key limitations for the currently available injectable hemostatic hydrogels. ${ }^{14}$ The delay of wound healing due to the

\footnotetext{
${ }^{a}$ State Key Laboratory of Polymer Physics and Chemistry, Changchun Institute of Applied Chemistry, Chinese Academy of Sciences, Changchun 130022, P. R. China. E-mail: shiqiang@ciac.ac.cn; Fax: +86431 85262126; Tel: +8643185262388

${ }^{b}$ College of Materials Science and Chemical Engineering, Harbin Engineering University, Harbin, 150001, P. R. China. E-mail: xuxiaodong@hrbeu.edu.cn

${ }^{c}$ School of Textile and Material Engineering, Dalian Polytechnic University, Dalian 116034, P. R. China

$\dagger$ Electronic supplementary information (ESI) available: Fig. S1-S7. See DOI: 10.1039/d1ma00137j
}

irregular loss of the wound also leads to an increase in mortality. ${ }^{15}$ For example, the cyanoacrylate-based tissue adhesive has the risk of inhibiting collagen remodeling and causing inflammation in the wound. ${ }^{16}$ Thus, it is extremely desired to develop injectable and biocompatible hydrogels with high capability for hemostasis and wound healing.

Recently, various injectable hemostatic hydrogels have been developed based on synthetic polymers (polycyanoacrylate, polyethylene glycol, polyurethane and polyester) and naturally derived polysaccharides (chitosan, cellulose, hyaluronic acid, alginic acid, chondroitin, etc. .) ${ }^{17-25}$ Compared with synthetic polymers, natural polysaccharides possess excellent biocompatibility. Chondroitin sulfate (CS) is a sulfated glycosaminoglycan that is abundant in the extracellular matrix of human tissues. It has been reported that chondroitin sulfate can regulate cell functions, such as cell migration and receptor binding. And the CS-based hydrogels possess high wound healing ability and biological activity at the cellular level. ${ }^{26-31}$ From the point of view of effective hemostasis, serotonin is an ideal candidate because serotonin is a natural component in the human body and can simulate the natural coagulation pathway through platelet activation. During coagulation, serotonin is released from the activated platelets and induces the secretion of platelet granules from activated platelets inversely. The platelet granules contain various hemostatic factors including fibrinogen, the von Willebrand 
factor, platelet factor 4 and platelet factor $\mathrm{V}$, which facilitate fast hemostasis. In addition, serotonin is reported to decrease apoptosis and increase the cell survival significantly in human fibroblasts and neonatal keratinocytes, and the endogenous serotonin pathway contributes to regulating the skin wound healing process. ${ }^{14,32,33}$ Therefore, serotonin-chondroitin sulfate injectable hydrogels are expected to possess high biocompatibility, hemostatic performance and wound healing capability. To the best of our knowledge, serotonin-chondroitin sulfate injectable hydrogels have not been fabricated.

In this study, a new injectable in situ hydrogel based on serotonin and chondroitin sulfate is developed. To guarantee the proper cross-linking, degradability and non-toxicity, the enzyme-catalyzed cross-linking methods are used. Horseradish peroxidase (HRP) is an efficient and specific biocatalyst in horseradish that induces cross-linking to form a hydrogel in combination with $\mathrm{H}_{2} \mathrm{O}_{2} \cdot{ }^{34-36}$ This enzymatic cross-linking can be carried out under mild conditions and endows the hydrogel with injectability, adjustable gel kinetics and controlled mechanical properties. The functional evaluation of the chondroitin sulfate-serotonin (CSS) hydrogel system is carried out by establishing a mouse liver hemorrhage model and a mouse back injury model. In order to confirm the versatility of serotonin-modified chondroitin sulfate and promote wound healing remarkably, aloe vera powder (AVP) is added to the hydrogel system. Aloe powder is a kind of curdlan obtained from freeze-dried aloe gel, which can stabilize the collagen on the wound surface and resist inflammation. ${ }^{37-40}$ The performance of the CSS hydrogel system is tested by establishing a mouse liver hemorrhage model and a mouse back injury model. CSS hydrogels doped with aloe powder are demonstrated to possess excellent hemostatic and wound healing capability, which provides a new strategy for the design and development of hemostatic materials.

\section{Results and discussion}

The preparation process of the CSS polymer is shown in Fig. 1A. The serotonin-modified chondroitin sulfate polymer is obtained by activating the carboxyl group on CS with the catalysis of EDC and NHS and the subsequent amination reaction of the amino group on serotonin. The chemical structures of CS, serotonin and CSS are characterized by ${ }^{1} \mathrm{H}$ NMR (Fig. 1B). In contrast to CS and serotonin, a specific peak of CSS between 6.8 and $7.5 \mathrm{ppm}$ (indole groups in aromatic protons) confirms the successful combination of serotonin and the main chain of CS. ${ }^{14,41}$ Comparing the ${ }^{1} \mathrm{H}$ NMR spectrum of CSS to that of CS, two new signal peaks appear at $2.4 \mathrm{ppm}$ and $2.9 \mathrm{ppm}$, which are attributed to the methylene and methyl peaks of CS-NHS. In addition, the enhanced peaks between 3.2 and $3.4 \mathrm{ppm}$ are due to the methylene on serotonin. The peaks between 3.5 and $4.6 \mathrm{ppm}$ in the CSS spectrum represent the methyne, methylene, methyl, and hydroxyl groups on the main chain of chondroitin sulfate. Meanwhile, in the UV-visible absorption spectrum, a new

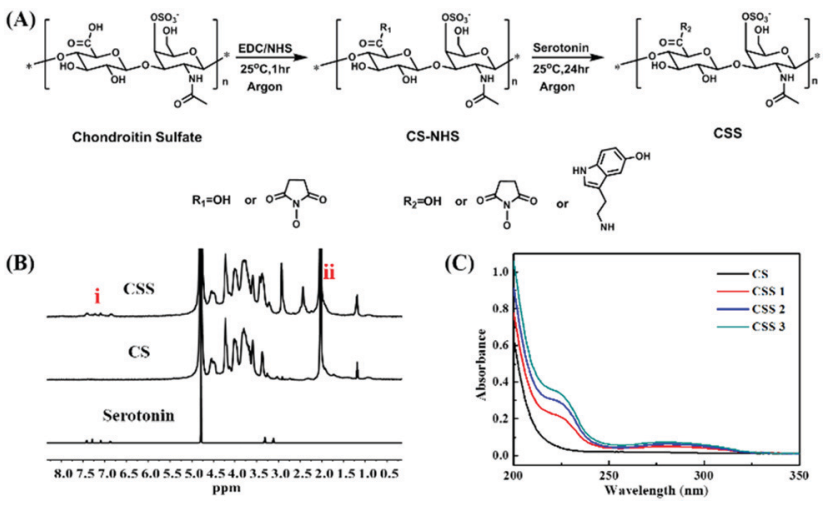

Fig. 1 (A) Synthesis pathway of the serotonin-chondroitin sulfate polymer (CSS); (B) ${ }^{1} \mathrm{H}$ NMR spectra of serotonin, CS, and CSS (from bottom upward); and (C) UV-visible (200-350 nm) spectra of CS, CSS 1, CSS 2, and CSS 3

absorption peak appears in the synthesized CSS polymer at a wavelength of $280 \mathrm{~nm}$, demonstrating the successful introduction of indole groups (Fig. 1C). The results show that the grafting rate of serotonin increases significantly with the enhancement of serotonin feed. The grafting reaction is further analyzed with FTIR spectra (ESI, $\dagger$ Fig. S1A). Compared with the CS spectrum, the new peaks at $\sim 1027 \mathrm{~cm}^{-1}$ and $\sim 1624 \mathrm{~cm}^{-1}$ in the CSS spectrum confirm the successful engraftment of the indole groups on CS.

The substitution degree of CSS with different feed molar ratios is determined by ${ }^{1} \mathrm{H}$ NMR spectroscopy (ESI, $\dagger$ Fig. S1B). By comparing the signal integral area of the aromatic proton peak in serotonin with that of the indicated proton in the CS main chain methyl ( $\sim 2.0 \mathrm{ppm})$, the DS values of indole groups are $8.0,13.0$ and $16.0 \%$. The three products with DS of 8.0, 13.0 and $16.0 \%$ are defined as CSS 1, CSS 2 and CSS 3 (Table 1). The higher the degree of substitution, the higher the content of serotonin bound on the CS main chain. Serotonin can not only be oxidized and cross-linked in the enzyme-catalyzed crosslinked hydrogel, but also acts with the nucleophile in the biomolecules to adhere to the tissue surface. Therefore, the product with the highest degree of substitution $(16.0 \%)$ is selected for the subsequent experiments.

CSS hydrogels are prepared by in situ crosslinking through HRP-mediated chemical reactions in the presence of $\mathrm{H}_{2} \mathrm{O}_{2}$ (Fig. 2A). The whole catalytic cycle is initiated by the interaction between $\mathrm{H}_{2} \mathrm{O}_{2}$ and the resting ferric state of HRP [Fe(III)], and then two single-electron reduction steps are performed to obtain two equivalent indole radicals. The generated indole radical forms intermolecular covalent linkages through the carbon-carbon bonds between the ortho-carbons of the

Table 1 Synthesis of CSS with different degrees of substitution (DS)

Theoretical feeding molar ratio

\begin{tabular}{llllc} 
No. & Samples & CS:EDC : NHS & CS:Serotonin & DS (\%) by ${ }^{1} \mathrm{H}$ NMR \\
\hline 1 & CSS1 & $1: 1: 1$ & $1: 1$ & 8 \\
2 & CSS2 & $1: 3: 3$ & $1: 3$ & 13 \\
3 & CSS3 & $1: 5: 5$ & $1: 5$ & 16
\end{tabular}


(A)

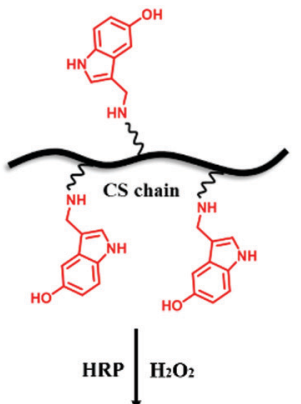

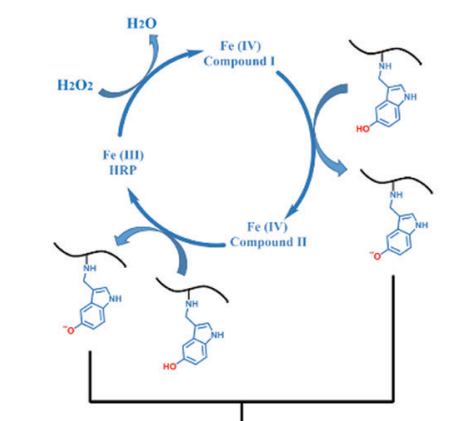

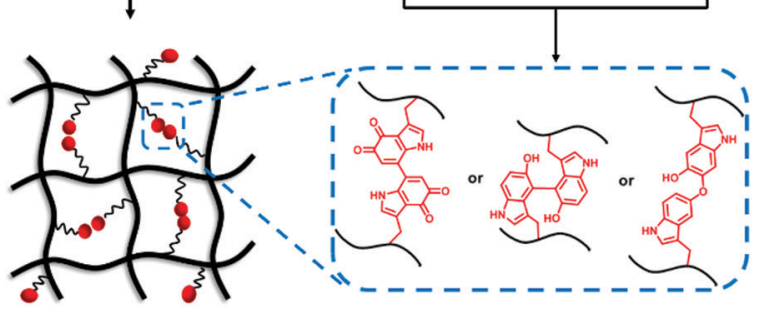

(B)

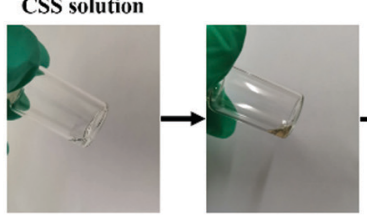

(C)

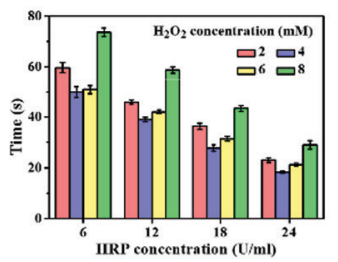

(D)

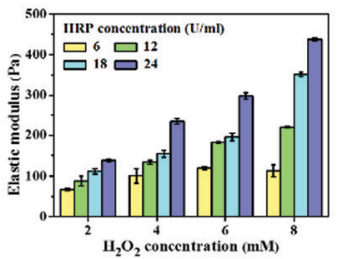

Fig. 2 (A) Schematic illustration of the CSS hydrogel formation and potential enzyme crosslinking mechanisms. (B) Color change of CSS hydrogels formed by enzymatic oxidation of CSS solution. (C) Gelation time of CSS hydrogels crosslinked using different concentrations of HRP and $\mathrm{H}_{2} \mathrm{O}_{2}(n=3)$. (D) Average elastic modulus $\left(G^{\prime}\right)$ of CSS hydrogels at different concentrations of $\mathrm{HRP}$ and $\mathrm{H}_{2} \mathrm{O}_{2}(n=3)$.

aromatic ring or through the carbon-oxygen bonds between the ortho-carbons and phenolic oxygen, thus preparing CSS hydrogels. ${ }^{42}$ A simple and efficient method is used to quickly form the CSS hydrogel by mixing $\mathrm{CSS} / \mathrm{H}_{2} \mathrm{O}_{2}$ and $\mathrm{CSS} / \mathrm{HRP}$ solution. By the oxidation of CSS polymer solution with HRP/ $\mathrm{H}_{2} \mathrm{O}_{2}$ enzyme within $1 \mathrm{~min}$, the color of the pre-gel solution immediately changes from colorless to light yellow, and finally to brown, indicating the sol-gel transition process due to the bonding between 5-hydroxyindole oxidation products in serotonin (Fig. 2B). In a complete catalytic cycle, fixing the concentration of HRP or $\mathrm{H}_{2} \mathrm{O}_{2}$ will generate a phenol free radical. The polymer network consists of phenolic compounds passing through the normal carbon on the aromatic ring and the phenolic oxygen. ${ }^{8,14}$ The cross-linking between carbon-carbon bonds or carbonoxygen bonds between positive carbon and phenolic oxygen forms the polymer network. Due to the substrate specificity and efficiency, mild reaction conditions and good cell compatibility, this cross-linking strategy is safe and suitable for biomedical applications.

In order to analyze the gel kinetics of CSS hydrogels, the gelation time of CSS polymer solutions with different concentrations of $\mathrm{HRP}$ and $\mathrm{H}_{2} \mathrm{O}_{2}$ is measured. Unless otherwise specified, the final optimized CSS polymer concentration for preparing CSS hydrogels is fixed at $5 \mathrm{wt} \%$. The gel time of the designed injectable hydrogel should meet the clinical needs within an appropriate range. When the concentration of $\mathrm{H}_{2} \mathrm{O}_{2}$ is lower than $2 \mathrm{mM}$, the hydrogel cannot be formed. When the concentration of $\mathrm{H}_{2} \mathrm{O}_{2}$ is higher than $8 \mathrm{mM}$, the gel time greatly increased, which does not meet our standard for preparing hydrogels. Therefore, the concentration of $\mathrm{H}_{2} \mathrm{O}_{2}$ is selected between $2 \mathrm{mM}$ and $8 \mathrm{mM}$ for the test. The concentration of $\mathrm{HRP}$ is selected in the range of $6 \mathrm{U} \mathrm{ml}^{-1}$ to $24 \mathrm{U} \mathrm{ml}^{-1}$. As shown in Fig. 2C, all CSS hydrogels are formed within $1 \mathrm{~min}$. When the concentration of $\mathrm{H}_{2} \mathrm{O}_{2}$ is fixed, the gelation time decreases significantly with the increase of HRP concentration because the high content of enzyme (HRP) can trigger more indole free radicals for effectively crosslinking the polymer. When the concentration of HRP is fixed, the gel time decreases with the increasing $\mathrm{H}_{2} \mathrm{O}_{2}$ level from $2.0 \mathrm{mM}$ to $4.0 \mathrm{mM}$, but when the concentration of $\mathrm{H}_{2} \mathrm{O}_{2}$ continues to increase, the gel time increases instead. A similar phenomenon has been observed in previous research, which was attributed to the reduction of the HRP activity with excessive $\mathrm{H}_{2} \mathrm{O}_{2}{ }^{36}$ The initial increase in $\mathrm{H}_{2} \mathrm{O}_{2}$ concentration can promote the cross-linking process, but the gel time will continue to increase under the influence of excessive $\mathrm{H}_{2} \mathrm{O}_{2}$. Compared with the wound healing CS-based hydrogels with good biocompatibility and hemostatic capacity, ${ }^{41}$ the advantage of CSS hydrogels in this work is that the gelling time is controllable and can be adapted in about $30 \mathrm{~s}$, which is necessary for the treatment of emergency bleeding.

The gel kinetics of CSS hydrogels are further analyzed using rheological tests (ESI, $\dagger$ Fig. S2). The frequency sweep test determines that the linear elastic region of the hydrogel is in the range of $0.1-10 \%$ strain. At this region, the storage modulus of the hydrogel is always higher than the loss modulus, confirming the stability of the obtained hydrogels (ESI, $\dagger$ Fig. S2A). ${ }^{43}$ The time for intersection of storage modulus and loss modulus is usually considered as the gel point, representing the transition of a viscous fluid from solution to gel. ${ }^{36}$ After this, the storage modulus increases rapidly with time and is always higher than the loss modulus, suggesting that the elastic behavior in the hydrogel system is dominant. As time goes by, the two moduli of the hydrogel finally reach a plateau, which indicates that gelation is complete and a stable hydrogel is formed (ESI, $\dagger$ Fig. S2B). The rheological results are generally consistent with the gelation time tested by the rotor stirring method.

The storage moduli of crosslinked hydrogels under various oxidation conditions are measured using a rheometer. As shown in Fig. 2D, the average storage modulus of CSS hydrogels increases proportionally with the increase of $\mathrm{H}_{2} \mathrm{O}_{2}$ and HRP concentrations, and the $\mathrm{H}_{2} \mathrm{O}_{2}$ concentration has a significant effect on the elastic behavior of the hydrogel. As the concentration of $\mathrm{H}_{2} \mathrm{O}_{2}$ and $\mathrm{HRP}$ increases, more indole radicals are oxidized for crosslinking reactions, resulting in a higher mechanical strength. 

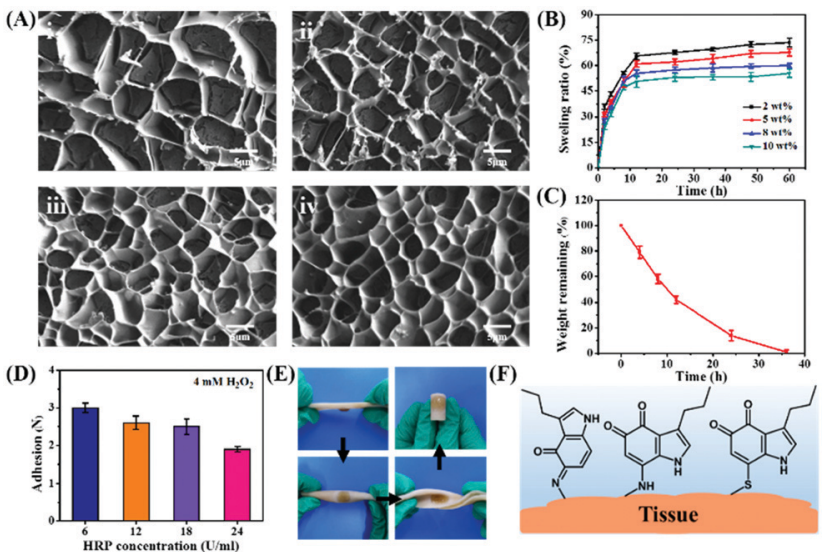

Fig. 3 (A) Microstructure of the crosslinked CSS hydrogels formed by using different concentrations of $\mathrm{HRP}$ at a fixed concentration of $\mathrm{H}_{2} \mathrm{O}_{2}$ (4 mM): (i) $6 \mathrm{U} \mathrm{ml}^{-1} \mathrm{HRP}$, (ii) $12 \mathrm{U} \mathrm{ml}^{-1} \mathrm{HRP}$, (iii) $18 \mathrm{U} \mathrm{ml}^{-1} \mathrm{HRP}$ and (iv) $24 \mathrm{U} \mathrm{ml}^{-1}$ HRP. (B) Measurement of swelling properties of CSS hydrogels formed with different concentrations of pre-gel solution upon incubation in PBS ( $\mathrm{pH} 7.4)$ at $37^{\circ} \mathrm{C}(n=3)$. (C) Enzymatic degradation profile of the CSS hydrogel formed with $18 \mathrm{U} \mathrm{ml}^{-1} \mathrm{HRP}$ and $4 \mathrm{mM} \mathrm{H}_{2} \mathrm{O}_{2}$ by chondrosulphatase treatment $(n=3)$. (D) Adhesive force of CSS hydrogels formed using different concentrations of HRP at a fixed concentration of $\mathrm{H}_{2} \mathrm{O}_{2}$ (4 mM). (E) Photographs of the hydrogels adhered to skin tissues were observed under torsion. (F) Schematic illustration of the chemistry for tissue adhesiveness of the CSS hydrogel.

The microstructure of CSS hydrogels is analyzed using a scanning electron microscope (SEM). As shown in Fig. 3A, the hydrogel has a porous structure with an irregular shape, which is conducive to absorbing excess exudates on the wound surface and increasing the concentration of red blood cells and platelets at the wound to accelerate blood clotting. In addition, the porous structure speeds up wound healing through facilitating cell migration and proliferation, nutrients supply and waste removal. Due to the increased crosslinking density, the internal pores of the hydrogel shrink with the increase of HRP concentration. Swelling degree reflects the interaction between the solution and the hydrogel, the structure of hydrogel and the degree of internal crosslinking. The swelling capacity of the CSS hydrogel is calculated by the mass change of the initial dry gel and the wet gel after being placed in PBS buffer ( $\mathrm{pH}$ 7.4) for a certain time. When the CSS polymer concentration is constant, the swelling degree of the CSS hydrogel increases continuously with the elongation of the incubation time, and the swelling equilibrium is reached in about 12 hours. When the CSS polymer concentration increases from $2 \mathrm{wt} \%$ to $10 \mathrm{wt} \%$, the equilibrium time is about $12 \mathrm{~h}$, but as the CSS polymer concentration increases, the equilibrium swelling degree of the hydrogel decreases from $68 \%$ to $48 \%$ (Fig. 3B). The high swelling hydrogel can effectively adsorb exudate from the serum to concentrate coagulation factors and cells, thereby accelerating coagulation at the wound site. ${ }^{44}$

The biodegradability of the hydrogel is related to its composition, physicochemical properties and physiological conditions. The hydrogel formed in $5 \mathrm{wt} \% \mathrm{CSS}$ polymer solution is used for enzymatic degradation by $0.01 \mathrm{U} \mathrm{ml}^{-1}$ chondroitinase. With the decomposition of the CS backbone by chondroitinase, the content of the hydrogel continues to decrease, confirming the biodegradability of CSS hydrogels. The CSS hydrogel is completely degraded by chondroitinase at about 36 hours (Fig. 3C), indicating that the CSS hydrogel can be removed naturally in the body after hemostasis is achieved.

The adhesion force of the CSS hydrogel is measured using a rheometer through an adhesion separation experiment. When the concentration of $\mathrm{H}_{2} \mathrm{O}_{2}(4 \mathrm{mM})$ is fixed, the adhesion force decreases with the increase of HRP concentration (Fig. 3D). The faster oxidation rate due to the increasing ratio of enzyme (HRP) to substrate $\left(\mathrm{H}_{2} \mathrm{O}_{2}\right)$ accelerates the internal crosslinking reactions between the conjugated serotonin molecules and CS. ${ }^{14}$ This may lead to the decreased interactions between the oxidized serotonin and other substrates, resulting in the reduced adhesion. The hydrogel is formed in situ on the surface of the pig skin, and the hydrogel adheres stable on the skin even the pig skin is bent, stretched and inverted (Fig. 3E). The stable adhesion of CSS hydrogels to tissues is due to the fact that serotonin derivatives (including serotonin free radicals and tryptamine diketones produced during serotonin oxidation) can bind to protein molecules containing amines, thiols, and phenols (Fig. 3F). ${ }^{45}$ Therefore, the physical and chemical properties of hydrogels, such as gelation time, modulus, swelling degree, degradation rate and adhesion strength, can be adjusted by tuning the concentration of the hydrogel pre-polymer solution and enzyme solution. Based on these results, CSS hydrogels cross-linked with $4 \mathrm{mM} \mathrm{H}_{2} \mathrm{O}_{2}$ and $18 \mathrm{U} \mathrm{ml}^{-1} \mathrm{HRP}$ are confirmed as the suitable adhesive for the subsequent experiments and further applications.

The hemolysis rate is the index for the toxicity of biomaterials to the red blood cells. ${ }^{46}$ The hemolysis rates of hydrogels are lower than 3\%, confirming that the CSS hydrogel system has no toxic effect on erythrocytes (Fig. 4A). The cell compatibility of CSS hydrogels is evaluated with L929 cells through CCK-8 cytotoxicity experiments. Compared with the control group, the cell survival rate of the hydrogel group remains above 90\% (Fig. 4B), exhibiting the high cytocompatibility. In addition, L929 cells showed significant proliferation after incubation in hydrogel extracts for 1 to $3 \mathrm{~d}$. As shown in Fig. 4C, the stain on the cell cytoskeleton further supports that the hydrogels are beneficial for cell viability and proliferation. The number of live/dead cells was counted using the confocal fluorescence images (live cells: green fluorescence and dead cells: red fluorescence), and no red fluorescence is seen in the image (Fig. S4, ESI $\dagger$ ). The results showed that the growth and proliferation of cells cultured with the CSS hydrogel extract were better. This phenomenon may be related to the nutritional properties of chondroitin sulfate, the skeleton material of the CSS hydrogel. It has previously been reported that chondroitin sulfate not only alleviates arthritic disease, but also promotes cell migration and speeds up metabolism. ${ }^{47}$

The adsorption capacity and porous structure of the hydrogels provide active sites for the adhesion and aggregation of blood cells, facilitating the thrombus formation. To confirm the induced adhesion of platelets and red blood cells on the CSS hydrogel, 
(A)

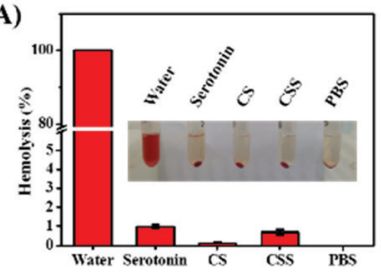

(C)

Control
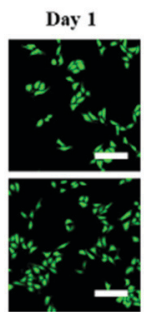

(B)

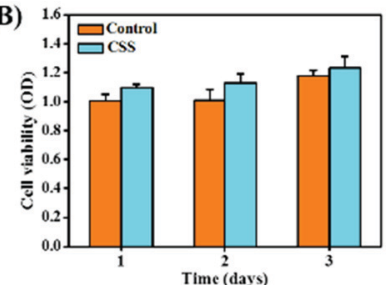

Day 2

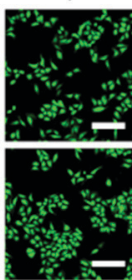

Day 3

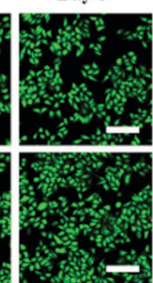

Fig. 4 (A) Hemolysis assay. Inset is the photograph of hemolytic red blood cells (RBCs) caused by the polymer and hydrogels. (B) Cell viability of L929 murine fibroblasts after incubation with hydrogel extracts for 1, 2, and 3 days. (C) Fluorescence images of live/dead stained L929 cells after incubation with the hydrogel extracts for 1, 2, and 3 days (scale bar: $100 \mu \mathrm{m}$. Cells cultured with DMEM as the control).

SEM is used to analyze blood cell adhesion (Fig. 5A). A large number of blood cells adhered to the surface of the CSS hydrogel. The platelets on the hydrogel are activated with spiny pseudopods (Fig. 5Ai). And the red blood cells adhered to the hydrogel gather together in an irregular shape. The normal red blood cells are biconcave disk-shaped, and most of the red blood cells are deformed after being incubated with the hydrogel (Fig. 5Aii). The blood clotting index (BCI) is determined to evaluate the
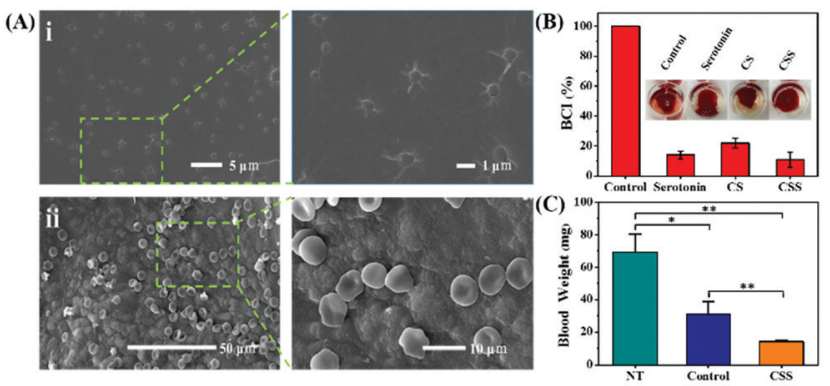

(D)

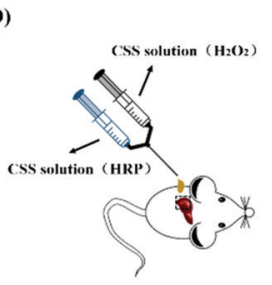

(E)

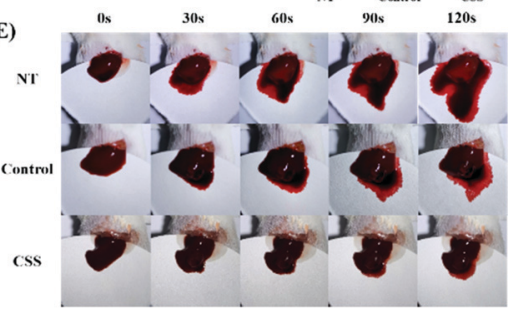

Fig. 5 (A) SEM images of RBCs and platelets adhesion on the hydrogels. (B) Blood clotting index ( $\mathrm{BCl})$. Inset is the photograph of an uncoagulated blood cell ruptured in water. (C) Total blood loss from the damaged livers at $120 \mathrm{~s}$ treated with hydrogels, commercial gum (control, positive control), and without treatment (NT, negative control) $\left(n=5,{ }^{*} p<0.05\right.$, ${ }^{* *} p<0.01$ ). (D) Schematic illustration of the mouse liver hemorrhage model. (E) Gross view of the bleeding mouse liver treated with hydrogels, commercial gum (control), and untreated (NT) every $30 \mathrm{~s}$ for $2 \mathrm{~min}$. hemostatic performance of the hydrogel in the presence of recalcified blood (Fig. 5B). Because the low BCI indicates high clotting capability, the BCI of the CSS hydrogel system is much lower than that of the control group, confirming the high hemostatic capability. Serotonin, a natural component of the body, activates platelets and releases clotting factors that cause blood to clot. CS forms a viscous substance in water-based solvents, thus playing the role of making blood become sticky and accelerating coagulation. As important components of CSS hydrogels, they play a key role in the adhesion of platelet and red blood cell experiments and BCI experiments.

To evaluate the hemostatic ability in vivo, a mouse liver hemorrhage model is set with an $18 \mathrm{G}$ needle puncture, and photographs of the bleeding site are taken every $30 \mathrm{~s}$ to monitor liver bleeding (Fig. 5C-E). At the same time, the weight of blood absorbed on the filter paper is used to measure liver bleeding until complete hemostasis. In this study, the group without treatment is set as a negative control, and a commercially available chitosan-gelatin hemostatic agent (Cofoe) as a positive control group. Serotonin and CS as the main components of hydrogels play an important role, so they are also compared as controls. The bleeding amounts are calculated at the end of hemostasis after $120 \mathrm{~s}$ (Fig. 5C). The bleeding amount for the CSS hydrogel is $14.2 \pm 0.8 \mathrm{mg}$, whereas those of the commercial glue and the negative control group are $31.0 \pm 7.7 \mathrm{mg}$ and $69.2 \pm 11.0 \mathrm{mg}\left({ }^{*} p<0.05,{ }^{*} p<0.01\right)$. With the same amount of the hemostatic agent, the hemostatic performance of CSS hydrogels is much better than that of the chitosan-gelatin hydrogel. Compared with the $120 \mathrm{~s}$ of the commercial glue to stop bleeding, the hemostatic time of the CSS hydrogel is about $30 \mathrm{~s}$ (Fig. 5E). Only serotonin or CS cannot be prepared into hydrogels and the two components alone have only a slight effect on the hemostatic properties (ESI, $\dagger$ Fig. S5). After the bleeding test, the liver tissues removed from each group are histologically analyzed with H\&E staining. Compared with normal liver tissue or the untreated group, the CSS hydrogel treatment area does not show any abnormal immune response, confirming the safety and biocompatibility of the medical CSS hydrogel (ESI, $\dagger$ Fig. S6).

The above results are consistent with the previous studies on the serotonin-induced hydrogel system. ${ }^{14}$ The CSS hydrogel can prevent blood loss by quickly cross-linking and sealing the bleeding site on the wound. Because of its porous structure, it can capture the exudate from the wound site, gather the coagulation factors in the blood around the wound, and enhance the natural coagulation effect by activating platelets and red blood cells. In addition, the oxidized indole group on serotonin can further undergo Michael addition and Schiff base reactions with amine, thiol and imidazole residues in extracellular matrix proteins and carbohydrates, so that the hydrogel can firmly adhere to the wound to achieve sealing and hemostasis. $^{48,49}$

The excellent biocompatibility, hemostasis and cell regulation render the CSS hydrogels available for wound healing. To prove the versatility of the CSS hydrogel and further improve the wound healing capability, the aloe vera powder (AVP) is added to the hydrogel system to obtain CSS-AVP hydrogels (the mass ratio of 

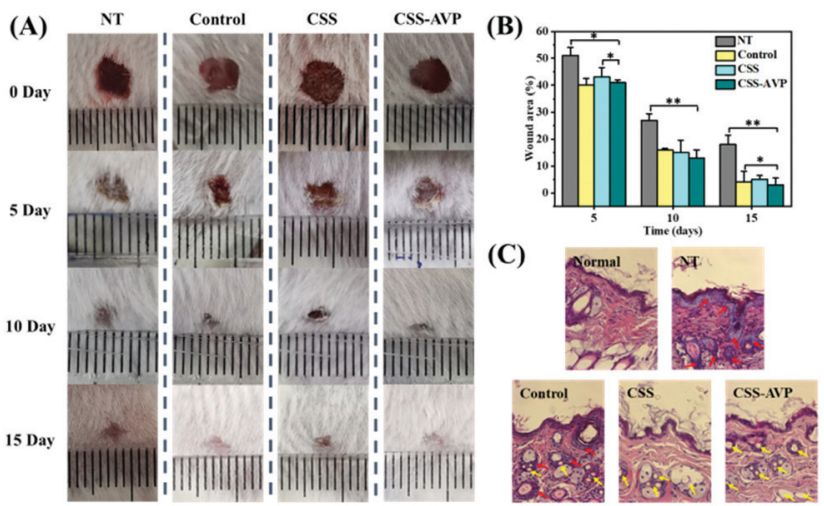

Fig. 6 (A) Photographs of wounds treated with CSS, CSS-AVP hydrogels and commercial dressing (control, positive control), and untreated (NT, negative control) at $0,5,10$, and 15 days. (B) Wound area percentage of rats treated by CSS, CSS-AVP hydrogels and commercial dressing (control), and untreated changed with time $\left(n=5,{ }^{*} p<0.05,{ }^{* *} p<0.01\right)$ (C) Histological analysis (H\&E staining) of the skin tissue 15 days postsurgery with the commercial adhesive (as the control), CSS and CSS-AVP hydrogels (inflammation, red arrow and blood vessels, yellow arrow).

the aloe vera powder to the CSS polymer was 1:5). The structure of the hydrogel is analyzed by infrared spectroscopy. The typical peaks of the $\mathrm{C}=\mathrm{C}$ group and $\mathrm{C}-\mathrm{N}$ group are observed at $\sim 1732 \mathrm{~cm}^{-1}$ and $\sim 1029 \mathrm{~cm}^{-1}$ in the infrared spectroscopy (ESI, $\dagger$ Fig. S7), confirming the successful doping of AVP. As shown in Fig. 6A, compared with the untreated group and control group (commercial dressings), CSS and CSS-AVP treated wounds exhibit high healing effects because the hydrogel dressings can absorb the wound exudate, prevent wound dehydration, and moisturize the wound at the same time to promote healing. On the fifth day after the operation, the naked eye image shows that the CSS and CSS-AVP hydrogel-treated wound has obvious regeneration of the epidermal cells, while the wound area of the control group reduces slightly. The wound areas of the CSS-AVP hydrogel, control group and untreated group are 43\%, 40\% and 51\%, respectively (Fig. 6B). The defects of the CSS hydrogel treatment basically recover on the tenth day. The new skin tissues after treatment are stained with H\&E and observed (Fig. 6C). Granulation tissue proliferation without inflammation (red arrow), larger blood vessel formation (yellow arrow) and higher collagen content appear in the wounds after treatment with CSS and CSS-AVP hydrogels, which is consistent with the morphology of normal tissue. In contrast, the untreated group and the commercial adhesive group show some degrees of inflammation. Thus, compared with the control group, CSS and CSS-AVP hydrogels exhibit reduced inflammation and enhanced number of blood vessels for repaired tissues.

This difference may be due to the perfect match and integration of the CSS hydrogel and tissue. After dropping the pre-gel solution into the injury site, the abundant indole groups in the polymer network possess strong binding affinity to various nucleophiles (for example, amino bonds, thiols and amines) on the tissue surface. CSS and CSS-AVP hydrogels provide the bionic microenvironment for cell proliferation and migration and accelerate the growth of new epidermis. ${ }^{27}$
In addition, aloe vera powder has been reported to contain many physiological active substances, which have antiinflammatory, immunomodulatory and promoting wound healing, etc. ${ }^{39}$

\section{Conclusions}

Inspired by a platelet coagulation mediator, a new type of in situ cross-linked injectable hemostatic hydrogel was developed based on chondroitin sulfate and serotonin. The structural, physical and biological and hemostatic properties of the hydrogel were systematically characterized. It was demonstrated that serotonin acted as a crosslinker to form adhesive hydrogels and a blood clotting mediator for rapid hemostasis. CS adjusted cell behaviors and fates to facilitate wound healing. The serotoninconjugated chondroitin sulfate hydrogel exhibited improved hemostatic capability in vivo and rapid wound healing after hemostasis. In addition, the wound healing capability of the hydrogel was further improved with the aloe vera powder, confirming the versatility of the hydrogel system. Therefore, chondroitin sulfate-serotonin hydrogels show the potential for effective hemostasis and wound healing.

\section{Experimental}

\section{Materials}

Chondroitin sulfate (CS) was obtained from Hua Xia Chemical Reagent Co., Ltd (Chengdu, China). Hydrogen peroxide $\left(\mathrm{H}_{2} \mathrm{O}_{2}\right.$, $30 \mathrm{wt} \%$ ), horseradish peroxidase (HRP), 1-(3-dimethylaminopropyl)3-ethylcarbodiimide hydrochloride (EDC), and $N$-hydroxysuccinimide (NHS) were all purchased from Aladdin. Serotonin hydrochloride was purchased from Energy Chemical. Aloe Vera Powder (AVP) was purchased from Yunnan Wanlv Biological Co., Ltd. All the chemicals were of analytical grade and were used without further purification.

\section{Characterization}

The chemical structure of the newly synthesized CSS polymer was measured using proton nuclear magnetic resonance $\left({ }^{1} \mathrm{H}\right.$ NMR, Bruker Avance $\left.500 \mathrm{MHz}\right)$, Fourier transform infra-red (FT-IR) and ultraviolet-visible (UV-visible) light spectrophotometer at $280 \mathrm{~nm}$. CSS with different substitutions was dissolved in deuterated water and characterized using ${ }^{1} \mathrm{H}$ NMR spectra. The degree of substitution (DS) was determined by comparing the integral area ratio of the peak of serotonin to that of the methyl group of the CS backbone. The degree of substitution of CSS and their preparation parameters are illustrated in Table 1.

\section{Synthesis of the CSS polymer}

Chondroitin sulfate-serotonin (CSS) conjugates were synthesized by modifying serotonin on the CS backbone using EDC and NHS via a simple carbodiimide coupling reaction. Briefly, CS was dissolved in distilled water at a concentration of $1 \mathrm{mg} \mathrm{m}{ }^{-1}$. EDC and NHS were added to the CS solution at 
an equal molar ratio to $\mathrm{CS}$ and stirred for a few minutes at $\mathrm{pH}$ 5.0-6.0, after which a predetermined amount of serotonin hydrochloride was added. The reaction mixture was protected with nitrogen and stirred overnight at room temperature at the same $\mathrm{pH}$. After the reaction, the resultant mixture was dialyzed using a dialysis membrane with a $M_{\mathrm{W}}$ cut-off of $7 \mathrm{kDa}$ in phosphate buffer solution (PBS) for 2 days. The synthesized product was collected by lyophilization thereafter, and the polymer was stored at $4{ }^{\circ} \mathrm{C}$ until use. ${ }^{14}$

\section{Enzymatic crosslinking of CSS hydrogels and gelation kinetics}

As a typical example, the CSS hydrogels were prepared in situ in the presence of $\mathrm{H}_{2} \mathrm{O}_{2}$ via HRP crosslinking. The concentration of enzyme was selected based on gel time. Briefly, a series of CSS polymer solutions was prepared by dissolving CSS in phosphate buffer solution (PBS: $0.01 \mathrm{M}$ and $\mathrm{pH}$ 7.4), which was then divided into two equal parts. The polymer solution was mixed with equal volumes of $\mathrm{H}_{2} \mathrm{O}_{2}$ and $\mathrm{HRP}$, respectively, and then the two ingredients were gently mixed with a doubletube syringe to form an in situ hydrogel. ${ }^{8,33}$ To evaluate the effect of $\mathrm{H}_{2} \mathrm{O}_{2}$ and HRP on gel time, $5 \mathrm{wt} \%$ of polymer solution was mixed with different concentrations of $\mathrm{H}_{2} \mathrm{O}_{2}(2.0-8.0 \mathrm{mM})$ and HRP (6.0-24.0 $\mathrm{U} \mathrm{ml}^{-1}$ ) in glass vials containing a stirring bar. The gelation time of CSS hydrogels was determined when the stirring bar speed was rapidly reduced and stopped due to rheological changes.

\section{Rheological experiments and adhesive force measurements}

The rheological properties of the hydrogels were characterized using a model MCR 702 rheometer. ${ }^{14,36}$ The pre-gel solution containing CSS, $\mathrm{H}_{2} \mathrm{O}_{2}$ and HRP was in situ injected into the parallel plate (plate diameter $=25 \mathrm{~mm}$ and gap $=0.5 \mathrm{~mm}$ ) with a double-tube syringe, and silicone oil was dripped around the mixture to prevent evaporation of water from the hydrogel. The storage modulus $\left(G^{\prime}\right)$ and loss modulus $\left(G^{\prime \prime}\right)$ of the hydrogel were measured in a frequency sweep mode, and the linear viscoelastic zone of the hydrogel was determined by observing the changes of the two. The strain value $(1 \%)$ in the linear viscoelastic region of the hydrogel was selected as the strain in a time sweep mode, and the rheometer test frequency was $1 \mathrm{~Hz}$. The elastic modulus of the hydrogel was determined by calculating the average storage modulus of each hydrogel at $1 \mathrm{~Hz}$.

The adhesive force of the hydrogel was measured in a tackseparation mode by recording the detachment force of the hydrogel between the probe and base plate while pulling the probe at $-10 \mu \mathrm{m} \mathrm{s} \mathrm{s}^{-1}$. All rheological measurements were performed in triplicate.

\section{Morphology of the hydrogels}

The microstructures of CSS hydrogels were observed using scanning electron microscopy (SEM) at an acceleration voltage of $20.0 \mathrm{kV}$. Before examination, the hydrogel specimens were freeze-dried. Then, the dried hydrogel was cut into thin sections using a sharp blade in liquid nitrogen. The cross-section of the dried sample was gold-coated and viewed using a microscope.

\section{Swelling and degradation profiles of the CSS hydrogels}

To test the swelling behavior of the crosslinked CSS gel, the hydrogel sample was prepared by enzymatic crosslinking at $37{ }^{\circ} \mathrm{C}$ for $24 \mathrm{~h}$ to ensure complete crosslinking, and the initial weight $\left(W_{0}\right)$ of the dry hydrogel was measured after being freeze-dried. The dried hydrogel samples were immersed in PBS solution at $37{ }^{\circ} \mathrm{C}$ and the weight of the hydrogel at each time point was measured. ${ }^{50}$ In order to reduce errors brought about by PBS solution left on the gel surfaces, the residual PBS solution was roughly wiped off, and the sample was weighed again $\left(W_{t}\right)$. The swelling ratio was calculated using the following equation:

$$
\text { Swelling ratio }(\%)=\frac{W_{t}-W_{0}}{W_{0}} \times 100 \%
$$

To investigate the in vitro enzymatic degradation of the crosslinked CSS gel, the hydrogel was incubated at $37{ }^{\circ} \mathrm{C}$ in PBS solution for 1 day to reach their swelling equilibrium. The mass of the hydrogel after swelling is recorded as the initial mass $\left(W_{\mathrm{i}}\right)$. After this, the CSS gel samples were immersed in $10 \mathrm{ml}$ of PBS solution with and without $0.01 \mathrm{U} \mathrm{ml}^{-1}$ of chondroitin enzyme. The media were removed and changes in the weight of the remaining hydrogel $\left(W_{\mathrm{r}}\right)$ were measured at each time point $\left(2,4,8,12,24\right.$, and 36 hours after incubation). ${ }^{51}$ The remaining weights were calculated using the following equation:

$$
\text { Remaining weight }(\%)=\frac{W_{t}}{W_{\mathrm{i}}} \times 100 \%
$$

\section{In vitro biocompatibility evaluation}

In vitro hemolysis assay is a universal method to evaluate the blood compatibility of materials. According to previous reports, the method for the hemolytic activity assay is as follows: centrifuge the fresh blood at a speed of $1500 \mathrm{rpm}$ for 10 minutes, remove the supernatant, wash the precipitated red blood cells repeatedly with PBS solution according to the above method until the supernatant does not show red, collect the purified red blood cells and further dilute to the final concentration $5 \%(\mathrm{v} / \mathrm{v})$. The CSS hydrogel was placed on the bottom of the test tube and the red blood cell suspension was added dropwise, mixed gently, and incubated in a $37{ }^{\circ} \mathrm{C}$ constant temperature shaker for $1 \mathrm{~h}$. After incubation, all the samples were centrifuged at $2000 \mathrm{rpm}$ for $5 \mathrm{~min}$. The obtained supernatants were transferred into a 96 well clear plate. The absorbance of the solutions at $540 \mathrm{~nm}$ was read using an enzyme standard instrument. Water served as the positive control and PBS served as the negative control. The hemolysis percentage of the hydrogel was calculated using eqn (3):

$$
\text { Hemolysis }(\%)=\frac{A_{\mathrm{p}}-A_{\mathrm{b}}}{A_{\mathrm{t}}-A_{\mathrm{b}}} \times 100 \%
$$

Here, $A_{\mathrm{p}}$ represents the absorbance of the sample, $A_{\mathrm{t}}$ represents the absorbance of the positive control, and $A_{\mathrm{b}}$ represents the absorbance of the negative control.

The cytotoxicity of the CSS hydrogel in vitro was evaluated by an indirect contact method, according to the ISO10993 
standard test that involves the L929 mouse fibroblasts being cultured with the hydrogel extracts. All the pre-gel solutions were sterilized by filtration via $0.22 \mu \mathrm{m}$ syringe filters in advance. After the hydrogels were formed in situ for $24 \mathrm{~h}$, the gel surfaces were washed with sterile PBS solution three times. Subsequently, the disinfected hydrogel samples were extracted in high glucose Dulbecco's Modified Eagle's Medium (DMEM) at a leaching ratio of $1 \mathrm{~cm}^{2} \mathrm{ml}^{-1}$ for 1 day. The fibroblasts were seeded at a density of $1.0 \times 10^{4}$ cells well $^{-1}$ in $200 \mu \mathrm{l}$ of medium containing $100 \mu \mathrm{l}$ DMEM (10 vol\% fetal bovine serum and $1.0 \mathrm{wt} \%$ penicillin-streptomycin) and $100 \mu \mathrm{l}$ sample extract and cultured at $37{ }^{\circ} \mathrm{C}$ in an incubator with $5 \% \mathrm{CO}_{2}$. The cell viabilities were tested by means of a CCK- 8 assay on day 1 , day 2 and day 3 .

To visually observe the cell viability of CSS gel culture, the cells of L929 cultured in the medium with and without CSS gel were stained with the live/dead viability Kit on day 1 , day 2, and day 3 , respectively. The viabilities of the staining cells were examined using a fluorescence microscope, and the ratio of viable cells (green) to dead cells (red) was quantified by manual counting from the acquired images.

\section{Whole blood clotting performance}

On the basis of previous reports, a whole blood clotting experiment was studied to analyze the coagulation ability of hydrogels. To eliminate the effect of blood clotting itself, fresh rabbit blood was collected using a vacuum tube containing a certain percentage of sodium citrate. Firstly, the hydrogels were prepared in situ in the middle of Petri dishes, and whole blood was added to the hydrogels, which started clotting by adding $0.2 \mathrm{M}$ of calcium chloride to the blood. Next, the hydrogels containing blood were incubated at $37{ }^{\circ} \mathrm{C}$ for a few minutes, then a certain amount of deionized water was slowly added along the edge of the plate. At this time, the red blood cells not wrapped in the clot were hemolyzed with water, and the solution was taken with a pipette and placed in a 96 well plate. Finally, the absorbance of free red blood cells in the solution was measured at $540 \mathrm{~nm}$. The control group was produced by dropping blood on the polystyrene pore plate. The blood clotting index (BCI) was calculated using eqn (4):

$$
\mathrm{BCI}=\frac{A_{\mathrm{s}}-A_{0}}{A_{\mathrm{c}}-A_{0}} \times 100 \%
$$

Here, $A_{\mathrm{s}}$ represents the absorbance of the sample, $A_{\mathrm{c}}$ represents the absorbance of the control, and $A_{0}$ represents the absorbance of deionized water.

\section{Blood cell and platelet adhesive performance}

The blood cell and platelet adhesive tests were conducted according to the literature. ${ }^{52,53}$ The whole blood was dropped on the hydrogel and then incubated at $37{ }^{\circ} \mathrm{C}$ for $30 \mathrm{~min}$. After incubation, the sample was washed with PBS ( $\mathrm{pH} 7.4$ ) solution 3 times followed by fixation in glutaraldehyde $(2.5 \%)$ for one night. Following this, the samples were washed with PBS and successively dehydrated in 20/40/60/80/100\% ethanol aqueous solution and dried in air. Red blood cells adhered to the surface of the hydrogel were observed using a scanning electron microscope (SEM). A similar protocol was followed to demonstrate cell adhesion on dressings. The platelet-rich plasma was isolated from the blood by centrifugation at $1500 \mathrm{rpm}$ for $10 \mathrm{~min}$. The hydrogel was then incubated in plasma at $37{ }^{\circ} \mathrm{C}$ for $30 \mathrm{~min}$ following the washing, fixation and dehydration treatment. Finally, the adhesion of platelet plasma was observed using SEM.

\section{In vivo hemostatic performance}

All animal procedures were performed according to the protocol approved by the Institutional Animal Care and Use Committee of China.

A mouse liver hemorrhage model was used to assess the hemostatic ability of CSS hydrogels in vivo. ${ }^{8}$ In short, twentyfive 4-week-old female mice were injected with an intraperitoneal anesthetic and an incision was made in the abdomen. Mice livers were exposed through an abdominal incision and a pre-weighed filter paper was placed under the liver. First, the tissue fluid around the liver is carefully removed to prevent inaccurate estimates of the amount of blood obtained by the filter paper. Liver bleeding is induced with an $18 \mathrm{G}$ needle and the damaged area is immediately covered with the CSS hydrogel or the commercial gel. After $2 \mathrm{~min}$, the filter paper with absorbed blood is weighed. No treatment after the liver was pricked with a needle was considered as a negative control, and commercial glue was used as a positive control. For statistical analysis, we used five mice for each experimental group $(n=5)$.

After completing the bleeding assessment, untreated mice with peritoneum and incision area closed with sutures were treated as a control group and the mice were sacrificed 3 days after treatment and their physiological status was observed.

\section{In vivo wound healing}

In order to study the ability of the hydrogel to promote wound healing, the full thickness infected skin defect models were established on the back of mice. ${ }^{54}$ Fifteen female mice ( 6 weeks, 150-200 g) were intraperitoneally anesthetized with $10 \mathrm{wt} \%$ chloral hydrate, and then their back hair was shaved with a surgical blade. The skin surface was disinfected with ethyl alcohol $(75 \%, \mathrm{v} / \mathrm{v})$, and two round full thickness wounds $\left(0.5 \times 0.5 \mathrm{~cm}^{2}\right)$ were established on the back of the mice. The wounds were placed about $2 \mathrm{~cm}$ from both sides of the mouse spine. The $5 \mathrm{wt} \%$ solid hydrogels and PBS (control) were applied to the wound, and the wounds of mice in each group were photographed $0,5,10$ and 15 days after the operation. Finally, on the 15th day, the mice were sacrificed, fresh wound tissues were cut off, and soaked in neutral paraformaldehyde for $\mathrm{H} \& \mathrm{E}$ staining. Image analysis software Image $\mathrm{J}$ was used to measure the size of the wound. The percentage of the wound area was calculated as follows:

$$
\text { Wound area }(\%)=\frac{A_{t}}{A_{0}} \times 100 \%
$$

Here, $A_{0}$ represents the area of the initial wound and $A_{t}$ represents the unclosed wound area when the mice were killed. 


\section{Histological analysis}

To investigate the inflammation on the wound surface, mice were sacrificed and liver tissues were taken for histological analysis 3 days after treatment of liver bleeding. Similarly, the mice were sacrificed 15 days after the skin healing treatment and the new skin tissue was taken for histological analysis. The collected samples were fixed with $4 \%$ paraformaldehyde and treated with a tissue processor, then embedded with paraffin, sliced into $5 \mu \mathrm{m}$ thick slices and stained with toluidine blue. Finally, all sections were analyzed and photographed by fluorescence microscopy.

\section{Conflicts of interest}

The authors declare no competing financial interest.

\section{Acknowledgements}

This work was financially supported by the National Natural Science Foundation of China (52061135202, 51573186, and 21807097), the National Key Research and Development Program of China (2018YFE0121400 and 2016YFC1100402), the Science Foundation of Jilin Province of China (20190701030GH) and the Open Research Fund of State Key Laboratory of Polymer Physics and Chemistry in CIAC, CAS (2020-19).

\section{Notes and references}

1 D. A. Hickman, C. L. Pawlowski, U. D. S. Sekhon, J. Marks and A. S. Gupta, Adv. Mater., 2018, 30, 1700859.

2 J. W. Simmons, J.-F. Pittet and B. Pierce, Curr. Anesthesiol. Rep., 2014, 4, 189-199.

3 F. K. Butler and L. H. Blackbourne, J. Trauma Acute Care Surg., 2012, 73, S395-S402.

4 X. Yang, W. Liu, Y. Shi, G. Xi, M. Wang, B. Liang, Y. Feng, X. Ren and C. Shi, Acta Biomater., 2019, 99, 220-235.

5 Y. Hu, Z. Zhang, Y. Li, X. Ding, D. Li, C. Shen and F. Xu, Macromol. Rapid Commun., 2018, 39, 1800069.

6 S. Lin, H. Yuk, T. Zhang, G. A. Parada, H. Koo, C. Yu and X. Zhao, Adv. Mater., 2016, 28, 4497-4505.

7 A. S. Hoffman, Adv. Drug Delivery Rev., 2012, 64, 18-23.

8 Y. Yao, Z. Xu, B. Liu, M. Xiao, J. Yang and W. Liu, Adv. Funct. Mater., 2020, 30, 2006944.

9 J. H. Ryu, Y. Lee, W. H. Kong, T. G. Kim, T. G. Park and H. Lee, Biomacromolecules, 2011, 12, 2653-2659.

10 Y. Yang, J. Zhang, Z. Liu, Q. Lin, X. Liu, C. Bao, Y. Wang and L. Zhu, Adv. Mater., 2016, 28, 2724-2730.

11 E. Lih, J. S. Lee, K. M. Park and K. D. Park, Acta Biomater., 2012, 8, 3261-3269.

12 N. Lang, M. J. Pereira, Y. Lee, I. Friehs, N. V. Vasilyev, E. N. Feins, K. Ablasser, E. D. O'Cearbhaill, C. Xu, A. Fabozzo, R. Padera, S. Wasserman, F. Freudenthal, L. S. Ferreira, R. Langer, J. M. Karp and P. J. del Nido, Sci. Transl. Med., 2014, 6, 218 ra6.

13 S. Hong, D. Pirovich, A. Kilcoyne, C.-H. Huang, H. Lee and R. Weissleder, Adv. Mater., 2016, 28, 8675-8680.
14 S. An, E. J. Jeon, J. Jeon and S.-W. Cho, Mater. Horiz., 2019, 6, 1169-1178.

15 S. Yan, T. Wang, L. Feng, J. Zhu, K. Zhang, X. Chen, L. Cui and J. Yin, Biomacromolecules, 2014, 15, 4495-4508.

16 A. J. Singer, J. V. Quinn and J. E. Hollander, Am. J. Emerg. Med., 2008, 26, 490-496.

17 M. Li, Y. Liang, J. He, H. Zhang and B. Guo, Chem. Mater., 2020, 32, 9937-9953.

18 J. Qu, X. Zhao, Y. Liang, T. Zhang, P. X. Ma and B. Guo, Biomaterials, 2018, 183, 185-199.

19 J. He, M. Shi, Y. Liang and B. Guo, Chem. Eng. J., 2020, 394, 124888.

20 N. Annabi, K. Yue, A. Tamayol and A. Khademhosseini, Eur. J. Pharm. Biopharm., 2015, 95, 27-39.

21 Y. Li, J. Rodrigues and H. Tomás, Chem. Soc. Rev., 2012, 41, 2193-2221.

22 P. J. M. Bouten, M. Zonjee, J. Bender, S. T. K. Yauw, H. van Goor, J. C. M. van Hest and R. Hoogenboom, Prog. Polym. Sci., 2014, 39, 1375-1405.

23 M. A. Boerman, E. Roozen, M. J. Sánchez-Fernández, A. R. Keereweer, R. P. Félix Lanao, J. C. M. E. Bender, R. Hoogenboom, S. C. Leeuwenburgh, J. A. Jansen, H. Van Goor and J. C. M. Van Hest, Biomacromolecules, 2017, 18, 2529-2538.

24 A. M. Behrens, N. G. Lee, B. J. Casey, P. Srinivasan, M. J. Sikorski, J. L. Daristotle, A. D. Sandler and P. Kofinas, Adv. Mater., 2015, 27, 8056-8061.

25 Z. Zeng, X.-M. Mo, C. He, Y. Morsi, H. El-Hamshary and M. El-Newehy, J. Mater. Chem. B, 2016, 4, 5585-5592.

26 W. Garnjanagoonchorn, L. Wongekalak and A. Engkagul, Chem. Eng. Process., 2007, 46, 465-471.

27 D.-A. Wang, S. Varghese, B. Sharma, I. Strehin, S. Fermanian, J. Gorham, D. H. Fairbrother, B. Cascio and J. H. Elisseeff, Nat. Mater., 2007, 6, 385-392.

28 H. D. Kim, E. A. Lee, Y.-H. An, S. L. Kim, S. S. Lee, S. J. Yu, H. L. Jang, K. T. Nam, S. G. Im and N. S. Hwang, ACS Appl. Mater. Interfaces, 2017, 9, 21639-21650.

29 Y. Liu, S. Wang, D. Sun, Y. Liu, Y. Liu, Y. Wang, C. Liu, H. Wu, Y. Lv, Y. Ren, X. Guo, G. Sun and X. Ma, Sci. Rep., 2016, 6, 29858.

30 S. Varghese, N. S. Hwang, A. C. Canver, P. Theprungsirikul, D. W. Lin and J. Elisseeff, Matrix Biol., 2008, 27, 12-21.

31 F. Chen, S. Yu, B. Liu, Y. Ni, C. Yu, Y. Su, X. Zhu, X. Yu, Y. Zhou and D. Yan, Sci. Rep., 2016, 6, 20014.

32 A. Sadiq, A. Shah, M. Jeschke, C. Belo, M. Qasim Hayat, S. Murad and S. Amini-Nik, Int. J. Mol. Sci., 2018, 19, 1034. 33 S. W. Whiteheart, Blood, 2011, 118, 1190-1191.

34 K. H. Bae and M. Kurisawa, Biomater. Sci., 2016, 4, 1184-1192.

35 Q. V. Nguyen, D. P. Huynh, J. H. Park and D. S. Lee, Eur. Polym. J., 2015, 72, 602-619.

36 W. Chen, R. Wang, T. Xu, X. Ma, Z. Yao, B. Chi and H. Xu, J. Mater. Chem. B, 2017, 5, 5668-5678.

37 T. Koike, H. Beppu, H. Kuzuya, K. Maruta, K. Shimpo, M. Suzuki, K. Titani and K. Fujita, J. Biochem., 1995, 118, 1205-1210. 
38 P. Chithra, G. B. Sajithlal and G. Chandrakasan, J. Ethnopharmacol., 1998, 59, 195-201.

39 R. Maenthaisong, N. Chaiyakunapruk, S. Niruntraporn and C. Kongkaew, Burns, 2007, 33, 713-718.

40 J. Hamman, Molecules, 2008, 13, 1599-1616.

41 H. Li, F. Cheng, X. Wei, X. Yi, S. Tang, Z. Wang, Y. Zhang, J. He and Y. Huang, Mater. Sci. Eng., C, 2021, 118, 111324.

42 J. W. Bae, J. H. Choi, Y. Lee and K. D. Park, J. Tissue. Eng. Regen. Med, 2014, 11, 1225-1232.

43 W. Han, B. Zhou, K. Yang, X. Xiong, S. F. Luan, Y. Wang, Z. Xu, P. Lei, Z. S. Luo, J. Gao, Y. J. Zhan, G. P. Chen, L. Liang, R. Wang, S. Li and H. Xu, Acta Biomater., 2020, 5, 768-778.

44 Y. Bu, L. Zhang, J. Liu, L. Zhang, T. Li, H. Shen, X. Wang, F. Yang, P. Tang and D. Wu, ACS Appl. Mater. Interfaces, 2016, 8, 12674-12683.

45 Y. Kato, J. Clin. Biochem. Nutr., 2016, 58, 99-104.

46 C. Che, L. Liu, X. Wang, X. Zhang, S. Luan, J. Yin, X. Li and H. Shi, ACS Biomater. Sci. Eng., 2020, 6, 1776-1786.
47 L. Lippiello, J. Woodward, R. Karpman and T. A. Hammad, Clin. Orthop. Relat. Res., 2000, 381, 229-240.

48 D. Duerschmied and C. Bode, Hamostaseologie, 2009, 29, 356-359.

49 A. L. Farré, J. Modrego and J. J. Zamorano-León, Horm. Mol. Biol. Clin. Invest., 2014, 18, 27-36.

50 H. Zhu, X. Mei, Y. He, H. Mao, W. Tang, R. Liu, J. Yang, K. Luo, Z. Gu and L. Zhou, ACS Appl. Mater. Interfaces, 2019, 12, 4241-4253.

51 I. Strehin, Z. Nahas, K. Arora, T. Nguyen and J. Elisseeff, Biomaterials, 2010, 31, 2788-2797.

52 S. S. Biranje, P. V. Madiwale, K. C. Patankar, R. Chhabra, P. Bangde, P. Dandekar and R. V. Adivarekar, Carbohydr. Polym., 2020, 239, 116106.

53 Y. Huang, X. Zhao, Z. Zhang, Y. Liang, Z. Yin, B. Chen, L. Bai, Y. Han and B. Guo, Chem. Mater., 2020, 32, 6595-6610.

54 L. Wang, X. Zhang, K. Yang, Y. V. Fu, T. Xu, S. Li, D. Zhang, L. N. Wang and C. S. Lee, Adv. Funct. Mater., 2019, 30, 1904156. 